\title{
Multiple Methods for Regional- to Mine-scale Targeting, Pataz gold field, northern Peru
}

\author{
W. K. WITT ${ }^{1}$, S. G. HAGEMANN ${ }^{1}$, J. OJALA ${ }^{2}$, C. LAUKAMP ${ }^{3}$, T. VENNEMANN ${ }^{4}$, C. \\ VILLANES ${ }^{5}$ AND V. NYKANEN ${ }^{6}$
}

${ }^{1}$ Centre for Exploration Targeting, University of Western Australia, WA 6009, Australia

${ }^{2}$ Store North Gull AS, PO Box 613, 971, Longyearbyen, Norway

${ }^{3}$ CSIRO Earth Science and Resource Engineering, 26 Dick Perry Avenue, Kensington, WA 6151, Australia

${ }^{4}$ University of Lausanne, $\mathrm{CH}-1015$, Lausanne, Switzerland

${ }^{5}$ Compania Minera Poderosa S.A., Av Primavera 834, Surco, Lima-33, Peru

${ }^{6}$ Geological Survey of Finland, PO Box 77, Fl-96101, Rovaneimi, Finland

\section{SUPPLEMENTARY PAPERS}

\author{
Australian Journal of Earth Sciences (2014) 61, 43-58. \\ http://dx.doi.org/10.1080/08120099.2013.763859
}

\begin{abstract}
* Appendix and Tables 1-5 [indicated in the text and listed at the end of the paper] are Supplementary Papers; copies may be obtained from the Geological Society of Australia's website (www.gsa.org.au), the Australian Journal of Earth Sciences website (www.ajes.com.au) or from the National Library of Australia's Pandora archive (http://nla.gov.au/nla.arc-25194).

†Corresponding author:_wittww@iinet.net.au
\end{abstract}

Table 1 Results of XRD analysis of samples from Vijus-Santa Filomena area.

Table 2 SEM analyses of white mica and clay minerals from mineralised quartzcarbonate-sulfide veins, Viju-Santa Filomena area.

Table 3 Results of stress modelling, Pataz district.

Table 4 SEM analyses of white mica and clay, batholith- hosted proximal alteration.

Table 5 Average and range of selected elements from 8 whole-rock geochemical samples of granodiorite from the Pataz district. 
Table 1 Results of XRD analysis of samples from Vijus-Santa Filomena area.

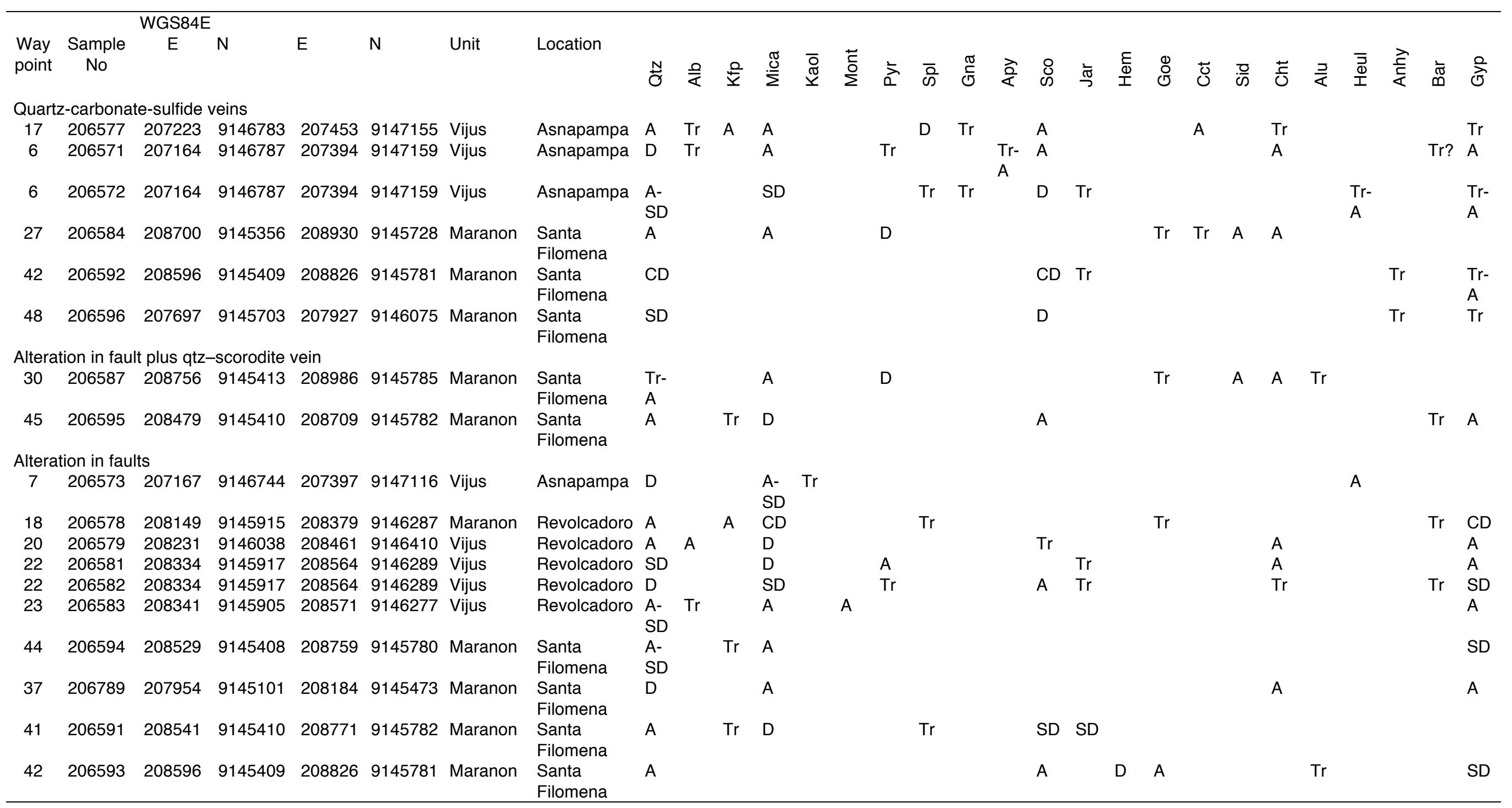


Witt et al. Supplementary Papers

Australian Journal of Earth Sciences 61, 43-58. http://dx.doi.org/10.1080/08120099.2013.763859

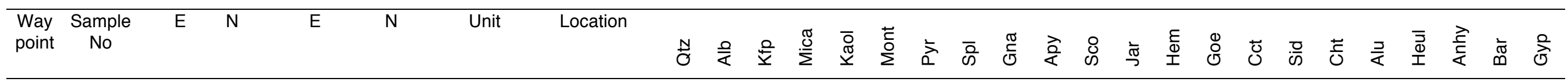

WGS84E

District-scale alteration

3920659020760891451912078389145563 Maranon Santa

Filomena

D SD A

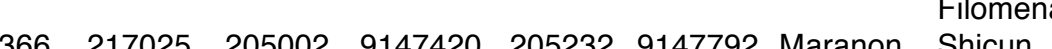

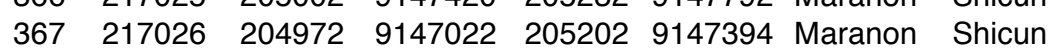

\begin{tabular}{llllll}
368 & 217027 & 204806 & 9146804 & 205036 & 9147176 \\
\hline
\end{tabular}

\begin{tabular}{llllll}
369 & 217028 & 204670 & 9146618 & 204900 & 9146990 \\
\hline
\end{tabular}

$370 \quad 217029204965 \quad 91465882051959146960$ Maranon Shicun

$371217030^{\star} \quad 20586791450312060979145403$ Maranon Vijus

D D $\operatorname{Tr}$ SD

SD SD A

D $\quad \mathrm{D} \quad \mathrm{Tr} \quad \mathrm{SD}$

CD $\quad$ CD $\operatorname{Tr}$ CD

$\begin{array}{llll}\text { SD } & \text { SD } & A & D \\ C D & C D & A & \text { CD }\end{array}$

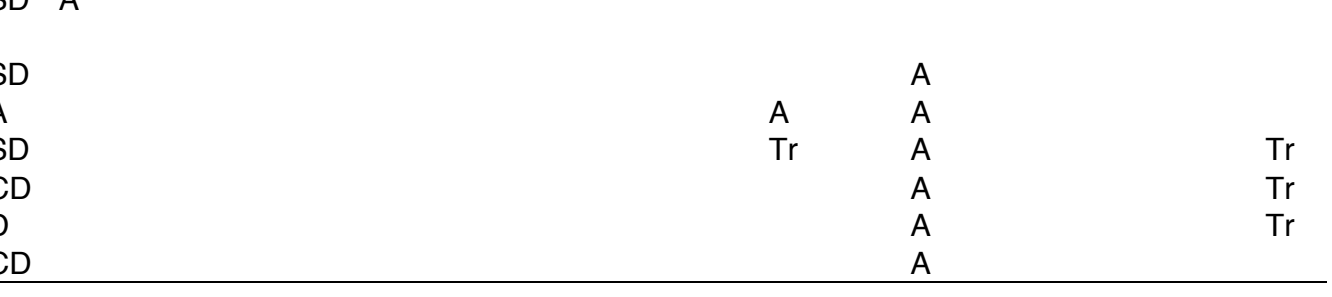

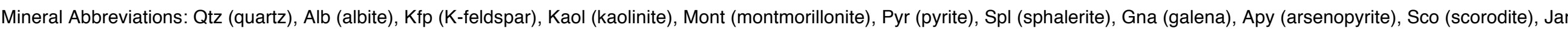
(jarosite), Hem (haematite), Goe (goethite), Cct (calcite), Sid (siderite), Cht (chlorite), Alu (alunite), Heul (heulandite), Anhy (anhydrite), Bar (barite), Gyp (gypsum)

XRD abundance codes: D (dominant), CD (co-dominant), SD (subdominant, $>20 \%$ ), A (accessory, 5 to $20 \%$ ), $\operatorname{Tr}$ (trace, $<5 \%$ ). 
Witt et al. Supplementary Papers

Australian Journal of Earth Sciences 61, 43-58. http://dx.doi.org/10.1080/08120099.2013.763859

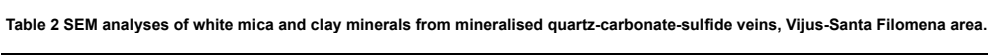

cations

cations

mol

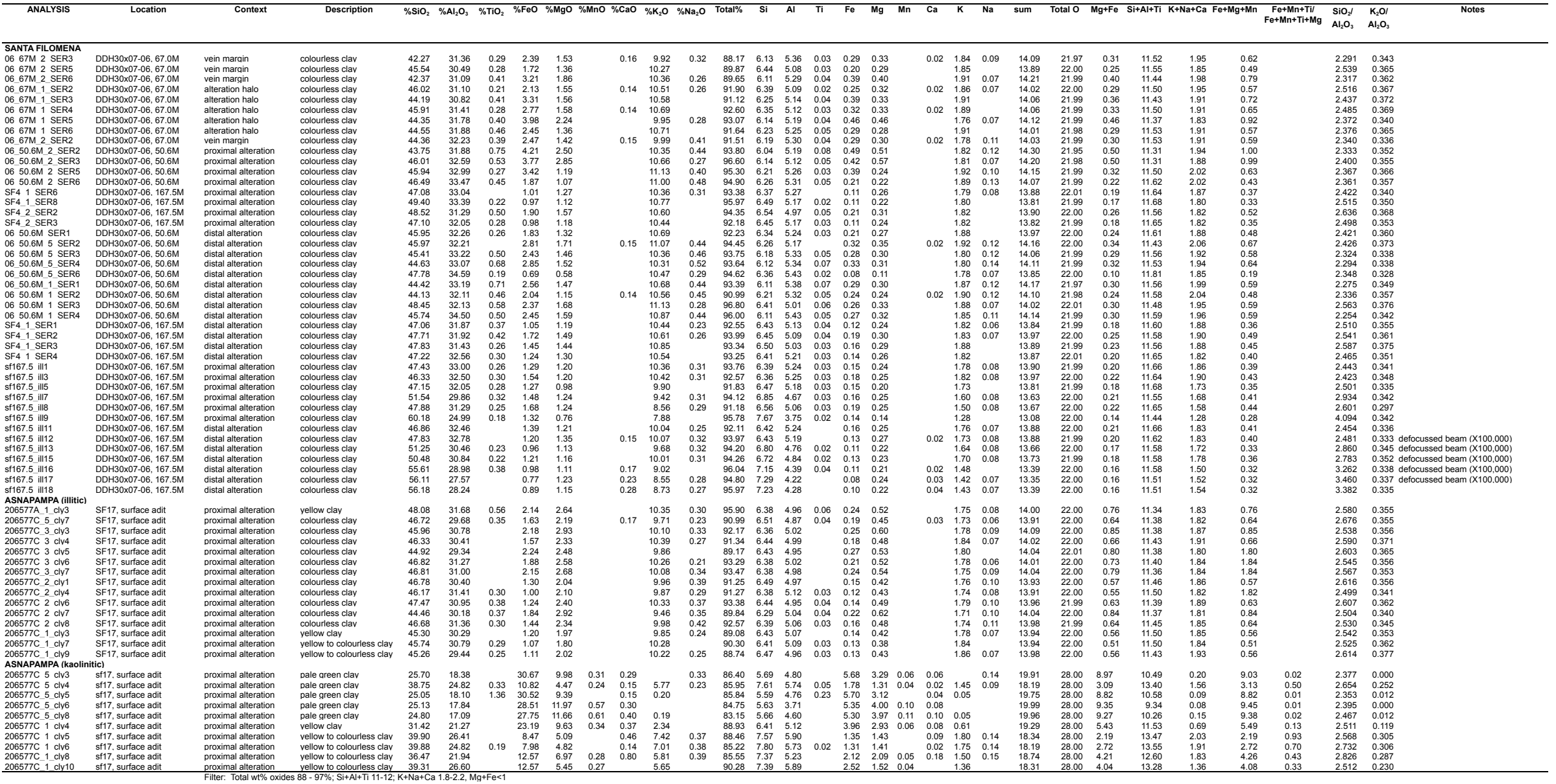

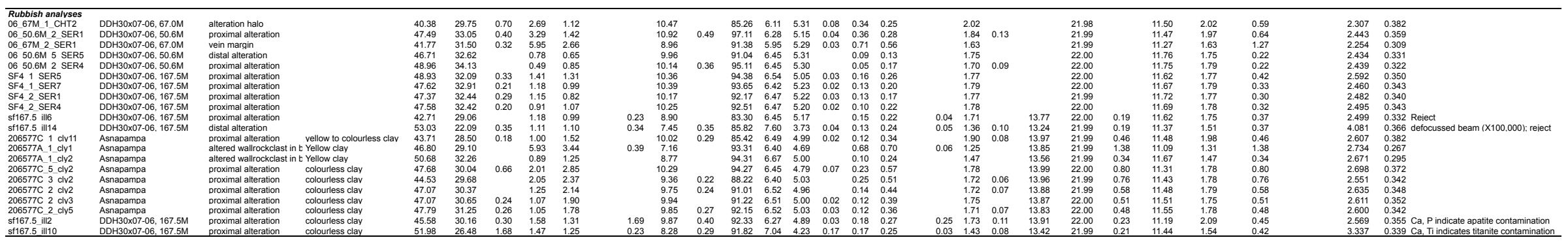


Table 3 Results of stress modelling, Pataz district.

\begin{tabular}{rrrrrrr}
\hline \multicolumn{2}{c}{ Lavasen not included } & \multicolumn{7}{c}{$\begin{array}{l}\text { Area } \\
\text { CLASS }\end{array}$} & \multicolumn{1}{c}{ SY From } & \multicolumn{1}{l}{ SY To } & \multicolumn{1}{c}{ km $^{2}$} & \multicolumn{1}{c}{ Area\% } & No_Points & Points\%* \\
\hline 6 & -69953480 & -69036568 & 24.5 & 3.9 & 143 & 19.1 \\
5 & -69980484 & -69953480 & 61.1 & 9.8 & 318 & 42.6 \\
4 & -70007489 & -69980484 & 607.2 & 97.5 & 701 & 93.8 \\
3 & -70034493 & -70007489 & 619.2 & 99.4 & 737 & 98.7 \\
2 & -70061497 & -70034493 & 621.1 & 99.7 & 742 & 99.3 \\
1 & -70856696 & -70061497 & 622.9 & 100.0 & 747 & 100.0
\end{tabular}

\begin{tabular}{|c|c|c|c|c|c|c|}
\hline \multicolumn{7}{|c|}{ Lavasen included } \\
\hline CLASS & SY From & SY To & $\begin{array}{l}\text { Area } \\
\text { km2 }\end{array}$ & Area\% & No_Points* & Points\%* \\
\hline 7 & -69931986.77 & -69037496 & 15.2 & 2.4 & 103 & 13.8 \\
\hline 6 & -69956657.93 & -69931986.77 & 28.4 & 4.6 & 178 & 23.8 \\
\hline 5 & -69981329.09 & -69956657.93 & 68.6 & 11.0 & 370 & 49.5 \\
\hline 4 & -70006000.25 & -69981329.09 & 600.7 & 96.4 & 695 & 93.0 \\
\hline 3 & -70030671.41 & -70006000.25 & 618.0 & 99.2 & 738 & 98.8 \\
\hline 2 & -70055342.57 & -70030671.41 & 620.7 & 99.6 & 742 & 99.3 \\
\hline 1 & -70852464 & -70055342.57 & 622.9 & 100.0 & 747 & 100.0 \\
\hline
\end{tabular}

${ }^{*}$ Vein abundance was determined by creating points at $100 \mathrm{~m}$ intervals along the veins and counting the number of points in a designated area. The cell size used for raster calculations was $25 \times 25 \mathrm{~m}$. Points that are closer than $25 \mathrm{~m}$ from each other were not counted twice. 
Witt et al. Supplementary Papers

Australian Journal of Earth Sciences 61, 43-58. http://dx.doi.org/10.1080/08120099.2013.763859

$\frac{\text { Table } 4 \text { SEM analyses of white mica and clay, batholith-hosted proximal alteration. }}{\text { Class }}$ Context

\begin{tabular}{|c|c|c|c|c|c|c|c|c|c|c|c|c|c|c|c|c|c|c|c|c|c|c|c|c|c|}
\hline NALY & & Location & Class & text & $\% \mathrm{SiO}_{2}$ & $\% \mathrm{Al}_{2} \mathrm{O}_{3}$ & $\% \mathrm{TiO}_{2}$ & & $\% \mathrm{Mg}$ & & $\% \mathrm{Ca}$ & $\% \mathrm{~K}_{2}$ & $\mathrm{Na}_{2} \mathrm{O}$ & $\begin{array}{llll}a 1 \% & \text { Si } & \text { Al }\end{array}$ & $\begin{array}{ll}\mathrm{Fe} & \mathrm{Mg} \\
\end{array}$ & ${ }^{\mathrm{Ca}}$ & 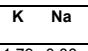 & $\begin{array}{l}\text { catio } \\
\text { sum } \\
\text { sum }\end{array}$ & Total $C$ & 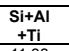 & $\begin{array}{c}\mathrm{K}+\mathrm{Na} \\
+\mathrm{Ca} \\
+\mathrm{aO}\end{array}$ & $\mathrm{Mg}+\mathrm{Fe}$ & $\begin{array}{l}\mathrm{Fe+Mg} \\
+\mathrm{Mn}\end{array}$ & 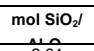 & 然 \\
\hline AFW $45 \mathrm{~W}$ & SER1 & $\begin{array}{ll}\text { Glortita; Glorita } \\
\text { Glorita Glorita }\end{array}$ & 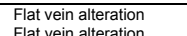 & $\begin{array}{l}\text { sericite } \\
\end{array}$ & 46.59 & 29.95 & & 3.56 & 1.88 & & 0.17 & 10.14 & 0.22 & $\begin{array}{lll}92.78 & 6.45 & 4.88\end{array}$ & 0.410 .39 & & & & 22.00 & 11.33 & $\frac{1.88}{1.88}$ & 0.80 & 0.80 & $\frac{1.64}{2.64}$ & 0.37 \\
\hline & $\begin{array}{l}R^{R} 2 \\
{ }^{2}\end{array}$ & 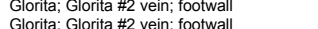 & vein & $\begin{array}{l}\text { sericite } \\
\text { socito }\end{array}$ & $\begin{array}{l}47.69 \\
474\end{array}$ & $\begin{array}{l}30.33 \\
30.34\end{array}$ & 025 & $\begin{array}{l}1.90 \\
1.20\end{array}$ & 1.80 1.80 & & 014 & $\begin{array}{l}10.84 \\
10.83\end{array}$ & & $\begin{array}{l}92.55 \\
92.55 \\
9.57 .91\end{array}$ & 0.220 .37 & 002 & 199 & $\begin{array}{l}13.95 \\
{ }^{13.95}\end{array}$ & 22:00 2200 & $\begin{array}{l}11.46 \\
{ }_{11} 441\end{array}$ & 1.90 & . 5.59 & 0.59 & 年, 267 & 0.39 \\
\hline & $\begin{array}{l}\text { toren3 } \\
\text {-SER4 }\end{array}$ & 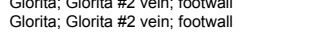 & $\begin{array}{l}\text { at vein alteration } \\
\text { at vein alteration }\end{array}$ & 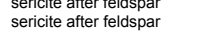 & $\begin{array}{l}4.41 \\
46.91\end{array}$ & $\begin{array}{l}22.444 \\
26.69\end{array}$ & 0.25 & 2.2 & $\begin{array}{l}1.85 \\
1.97\end{array}$ & & & $\begin{array}{l}11.23 \\
10.75\end{array}$ & 0.23 & 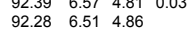 & $\begin{array}{l}0.26 \\
0.30\end{array}$ & 0.02 & 1.99 & $\begin{array}{l}14.06 \\
14.04\end{array}$ & .00 & .37 & $\begin{array}{l}2.01 \\
1.96\end{array}$ & .71 & 71 & $\begin{array}{l}2.14 \\
2.99\end{array}$ & $\begin{array}{l}0.41 \\
0.44\end{array}$ \\
\hline$N_{-3}-3$ & MUS1 & Glorita; Glorita \#2 vein, footwall & $\begin{array}{l}\text { tvein alteration } \\
\text { tvion }\end{array}$ & uscovite after biotite & 46.99 & 30.82 & 0.27 & 1.51 & 1.76 & & & 11.31 & & $\begin{array}{lllll}92.73 & 6.46 & 4.99 & 0.03\end{array}$ & $\begin{array}{lll}.17 & 0.36\end{array}$ & & 1.98 & 13.99 & 22.00 & 11.48 & 1.98 & 0.53 & 0.53 & 2.59 & 0.40 \\
\hline $\begin{array}{l}w_{-3}^{3} \\
w^{-3} \\
3\end{array}$ & 3 & 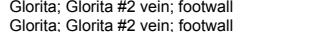 & $\begin{array}{l}\text { tivenin alteration } \\
\text { tvein nateration }\end{array}$ & $\begin{array}{l}\text { siccovite after biotite } \\
\text { siscovite after biotite }\end{array}$ & $\begin{array}{l}47.11 \\
46.99\end{array}$ & $\begin{array}{l}30.022 \\
30.06\end{array}$ & $\begin{array}{l}0.47 \\
0.34\end{array}$ & $\begin{array}{l}1.79 \\
1.90\end{array}$ & $\begin{array}{l}1.83 \\
1.84\end{array}$ & & 0.24 & $\begin{array}{l}11.15 \\
11.03\end{array}$ & & 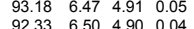 & 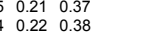 & 02 & & $\begin{array}{l}14.08 \\
11.03\end{array}$ & 2200 & $\begin{array}{ll}11.43 \\
11.44\end{array}$ & 2.07 & 0.58 & .58 & 2.64 & 0.40 \\
\hline & MUS4 & Vall & Iton & $\begin{array}{l}\text { muscov } \\
\text { muscov }\end{array}$ & & $\begin{array}{l}30.00 \\
30.05\end{array}$ & $\begin{array}{l}0.34 \\
0.39\end{array}$ & 1.8 & 1.83 & & & 111.36 & & & & & & $\begin{array}{l}14.02 \\
14.02\end{array}$ & & & & & & & $\begin{array}{l}0.40 \\
0.41\end{array}$ \\
\hline & -MUS1 & Glorita; Gorita \#2 vevin, footwall & $\begin{array}{l}\text { tein alteration } \\
\text { tven }\end{array}$ & muscovite after biotite & 47.19 & 30.15 & 0.51 & 1.94 & 1.90 & & & $\begin{array}{l}10.87 \\
10.87\end{array}$ & & 92.756 .504 .890 .05 & 0.220 .39 & & 1.91 & 13.96 & 22.01 & 11.44 & .91 & 61 & .61 & 2.66 & 39 \\
\hline & MUS2 & rita; G Glorita \#2 vein; footwall & $\begin{array}{l}\text { ein alteration } \\
\text { ention }\end{array}$ & $\begin{array}{l}\text { muscovite after biotite } \\
\text { muscosite atater biofitie }\end{array}$ & $\begin{array}{l}47.43 \\
4658\end{array}$ & $\begin{array}{l}29.85 \\
30.17\end{array}$ & $\begin{array}{l}0.43 \\
0.30\end{array}$ & $\begin{array}{l}1.85 \\
162\end{array}$ & 1.93 & & & $\begin{array}{l}11.37 \\
11.07\end{array}$ & & $\begin{array}{llll}92.96 & 6.52 & 4.84 & 0.04 \\
9136 & 649 & 496 & 0.03\end{array}$ & 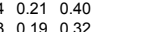 & & $\begin{array}{l}2.00 \\
197\end{array}$ & 96 & & 1148 & $\begin{array}{l}00 \\
07 \\
97\end{array}$ & & & 2.70 & 10 \\
\hline & US3 3 & 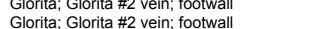 & $\begin{array}{l}\text { tveninateration } \\
\text { tvein atteration }\end{array}$ & $\begin{array}{l}\text { muscovite aft } \\
\text { muscovite aft }\end{array}$ & $\begin{array}{l}46.58 \\
46.42\end{array}$ & $\begin{array}{l}30.85 \\
29.85\end{array}$ & $\begin{array}{l}0.30 \\
0.31\end{array}$ & 52 & $\begin{array}{l}1.54 \\
1.73\end{array}$ & & & $\begin{array}{l}11.07 \\
11.00\end{array}$ & & 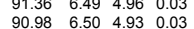 & & & $\begin{array}{l}1.97 \\
1.97\end{array}$ & & & $\begin{array}{l}11.48 \\
11.46\end{array}$ & & & & & 40 \\
\hline & 1_SER1 & Glorita; Glorita \#2 vein, footwall & at vein alteration & $\begin{array}{l}\text { sericite after feldspar } \\
\text { ser }\end{array}$ & 46.57 & .22 & & $\begin{array}{l}1.89 \\
1.86\end{array}$ & 1.84 & & & 10.46 & & $\begin{array}{lll}90.10 & 6.57 & 4.86\end{array}$ & 0.220 .39 & & $\begin{array}{l}1.98 \\
1.88\end{array}$ & $\begin{array}{ll}13.99 \\
13.92\end{array}$ & 99 & 11.48 & 1.88 & 61 & 0.61 & 2.71 & 39 \\
\hline & & Glorita; Glorita \#2 vein; footwall & at vein alteration & sericite after feldspar & 45.63 & 28.22 & & 1.90 & 1.77 & & 0.23 & 10.72 & & $\begin{array}{lll}88.71 & 6.56 & 4.78\end{array}$ & $0.23 \quad 0.38$ & 0.04 & & 13.96 & 21.98 & 34 & 2.01 & 0.61 & 0.61 & 2.75 & 0.41 \\
\hline & $\begin{array}{l}\text { 1-SER3 } \\
1-S R 24\end{array}$ & $\begin{array}{l}\text { Glorita; } \mathrm{G} \\
\text { Glorita: } \mathrm{G}\end{array}$ & $\begin{array}{l}\text { at vei } \\
\text { at vei } \\
\text { and }\end{array}$ & sericite & $\begin{array}{l}47.62 \\
47.01\end{array}$ & $\begin{array}{l}07 \\
14\end{array}$ & & & 1.9 & & & $\begin{array}{l}10.81 \\
1076\end{array}$ & $\begin{array}{l}0.25 \\
0.21\end{array}$ & 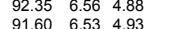 & 0 & & 0 & 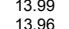 & 99 & 46 & 97 & 58 & 53 & 2.69 & 39 \\
\hline$=w$ & TSER & 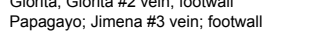 & $\begin{array}{l}\text { Falvevin alteratuon } \\
\text { Flat vein alteration }\end{array}$ & $\begin{array}{l}\text { crite aater efldsspar } \\
\text { iscovite after biotite }\end{array}$ & $\begin{array}{l}47.71 \\
47.76\end{array}$ & $\begin{array}{l}38.67 \\
28.67\end{array}$ & 0.80 & $\begin{array}{l}1.89 \\
1.60\end{array}$ & 1.8. & & & $\begin{array}{l}{ }_{10.68} \\
10.68\end{array}$ & & $\begin{array}{llll}91.60 & 6.53 & 4.93 & \\
91.53 & 6.64 & 4.70 & 0.08\end{array}$ & 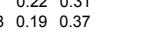 & & 1.8. & & .01 & 46 & 1.89 & 3 & 56 & $\begin{array}{l}2.65 \\
2.83\end{array}$ & 90 \\
\hline & 1-MUS2 & $\begin{array}{ll} & \\
\text { Papagayo; jit } & \text { jit }\end{array}$ & it vein alteration & $\begin{array}{l}\text { muscovite after biotitie } \\
\text { muscote }\end{array}$ & 45.08 & $\begin{array}{l}28.81 \\
2287\end{array}$ & 1.16 & 2.00 & 1.58 & & 0.20 & 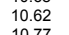 & & $\begin{array}{llll}89.48 & 6.45 & 4.86 & 0.12\end{array}$ & $0.24 \quad 0.34$ & 0.03 & 1.94 & 13.98 & .00 & 43 & 1.97 & .58 & 58 & 2.66 & 40 \\
\hline & & \#3 vein; footwall & & & $\begin{array}{l}46.97 \\
4682\end{array}$ & 28.72 & $\begin{array}{l}0.46 \\
0.27\end{array}$ & & 1.67 & & & $\begin{array}{l}10.77 \\
10.70\end{array}$ & & $\begin{array}{lllll}90.13 & 6.63 & 4.78 & 0.05\end{array}$ & & 0.02 & 1.94 & 92 & 00 & & & & & 78 & 41 \\
\hline$F W$ & $\begin{array}{l}\text { MUS4 } \\
\text { SER1 }\end{array}$ & 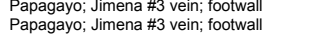 & $\begin{array}{l}\text { Flat vein alteration } \\
\text { Flat vein alteration }\end{array}$ & $\begin{array}{l}\begin{array}{l}\text { muscocivi } \\
\text { sericite a }\end{array} \\
\text { a }\end{array}$ & $\begin{array}{l}46.82 \\
45.01\end{array}$ & $\begin{array}{l}29.57 \\
29.54\end{array}$ & & $\begin{array}{l}1.36 \\
1.28\end{array}$ & $\begin{array}{l}1.76 \\
1.14\end{array}$ & & & 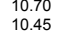 & 0.23 & $\begin{array}{l}90.86 \\
87.87 \\
8\end{array}$ & $\begin{array}{l}50.37 \\
0.25\end{array}$ & & $\begin{array}{lll}1.91 & 0.07\end{array}$ & 92 & 99 & .54 & 90 & 53 & 40 & $\begin{array}{l}2.69 \\
2.59\end{array}$ & 38 \\
\hline & $\begin{array}{l}\text { SER2 } \\
\text { SER }\end{array}$ & 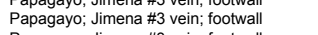 & $\begin{array}{l}\text { Flat vein alteration } \\
\text { Finn }\end{array}$ & $\begin{array}{l}\text { sericite after feldspsar } \\
\text { selspar }\end{array}$ & 45.61 & $\begin{aligned} 29.64 \\
29.61\end{aligned}$ & & 然 & 1.44 & & & 10.91 & $0.6-4=28$ & $\begin{array}{l}81.01 \\
89.75\end{array}-14$ & 0.210 .31 & & 1.98 & & 00 & 11.45 & .98 & 0.52 & 52 & 2.62 & \\
\hline & & ipagayo; Jimen & eration & $\begin{array}{l}\text { sericite af } \\
\text { secicite at }\end{array}$ & $\begin{array}{l}46.41 \\
4644\end{array}$ & 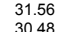 & & 1.08 & 1.06 & & & $\begin{array}{l}11.28 \\
11.12\end{array}$ & 025 & $\begin{array}{lll}91.31 & 6.46 & 5.18 \\
9146 & 6.47 & 5010\end{array}$ & 0.130 .22 & & 2.00 & 13.99 & $\begin{array}{l}22.01 \\
21.99\end{array}$ & $\begin{array}{l}11.64 \\
11.50\end{array}$ & & & 35 & $\begin{array}{l}2.50 \\
2.59\end{array}$ & \\
\hline & $\begin{array}{l}\text { SER4 } \\
\text { SER5 }\end{array}$ & $\begin{array}{l}\text { apagayo; Jime } \\
\text { apagay; Jime }\end{array}$ & $\begin{array}{l}\text { eratition } \\
\text { eration }\end{array}$ & eldspar & $\begin{array}{l}46.44 \\
45.75\end{array}$ & $\begin{array}{l}30.48 \\
30.17\end{array}$ & 0.17 & $\begin{array}{l}1.56 \\
1.62\end{array}$ & $\begin{array}{l}1.50 \\
1.28\end{array}$ & & & $\begin{array}{l}11.12 \\
11.25\end{array}$ & & 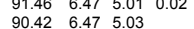 & $\begin{array}{l}0.18 \\
0.19 \\
0.27\end{array}$ & & $\begin{array}{l}1.98 \\
2.03\end{array}$ & & 00 & 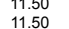 & & 46 & 49 & $\begin{array}{l}2.59 \\
2.58\end{array}$ & 40 \\
\hline & SER1 & $\begin{array}{l}\text { ppagayo; Imeri } \\
\text { pagarit }\end{array}$ & wv & (a) & 48.86 & 31.39 & 0.21 & $\begin{array}{l}1.02 \\
2.09\end{array}$ & 1.12 & & 0.17 & 11.09 & & $\begin{array}{lll}90.42 & 0.47 & 5.03 \\
95.42 & 4.93\end{array}$ & 20.230 .22 & 0.02 & $\begin{array}{l}2.08 \\
1.88\end{array}$ & 10.99 & 21.99 & 11.46 & .90 & 0.45 & .46 & 2.65 & 38 \\
\hline & SER2 & pagayo; Glorita vein & Wvein & fg sericite & 48.39 & 30.81 & 0.26 & 59 & 1.18 & & & 11.43 & & $\begin{array}{llll}94.67 & 6.54 & 4.91 & 0.03\end{array}$ & 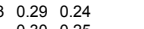 & & 1.97 & & 22.00 & 48 & 97 & 0.53 & & 2.67 & 0 \\
\hline & & $\begin{array}{l}\text { pagayo; Glorita vein } \\
\text { pagayo; Glorita vein }\end{array}$ & ein & $\begin{array}{l}\text { fg sericite } \\
\text { ff sericite }\end{array}$ & $\begin{array}{l}46.43 \\
48.09\end{array}$ & 29.42 & & & 125 & & & 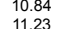 & & & 0. & & 1.99 & & & & & & & & \\
\hline & CRB2 & $\begin{array}{l}\text { pagayo, Glorita vein } \\
\text { pagayo; Glon }\end{array}$ & & $\begin{array}{l}\text { fg sericite } \\
\text { fons }\end{array}$ & $\begin{array}{l}40.09 \\
46.68\end{array}$ & $\begin{array}{l}28.07 \\
28.07\end{array}$ & .27 & & 1.24 & & & 10.64 & & $\begin{array}{l}89 \\
89\end{array}$ & 0.320 & & 1.94 & & & & & & & & \\
\hline & SER6 & pagayo; Glorita vein & Wvein & ff sericite & 46.99 & 29.87 & 0.28 & 2.63 & 1.14 & & 0.19 & 10.94 & & $\begin{array}{llll}92.42 & 6.51 & 4.88 & 0.03\end{array}$ & $\begin{array}{lll}3 & 0.30 & 0.24\end{array}$ & 0.03 & 1.93 & & 99 & 42 & 96 & .54 & 54 & 2.67 & \\
\hline & & $\begin{array}{l}\text { apagayo; Glorita vein } \\
\text { pagago: Glorit vevin }\end{array}$ & IW vein & fg sericite & & $\begin{array}{l}29.96 \\
33103\end{array}$ & 0.20 & & $\begin{array}{l}1.17 \\
1.13\end{array}$ & & & & & 90.71 & & & & & & & & & & & \\
\hline & 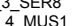 & $\begin{array}{l}\text { apagayayo; Gilonta velin } \\
\text { onsunulo; Consuelo vein }\end{array}$ & Wvein & $\begin{array}{l}\text { Ig serticie } \\
\text { muscovite }\end{array}$ & $\begin{array}{l}4.4 .42 \\
46.97\end{array}$ & $\begin{array}{l}33.03 \\
30.27\end{array}$ & 0.39 & & $\begin{array}{l}1.13 \\
1.39\end{array}$ & & & $\begin{array}{l}10.96 \\
10.96\end{array}$ & & $\begin{array}{l}92 . \\
91 .\end{array}$ & $\begin{array}{l}0.22 \\
40.130\end{array}$ & & 1 & & & & & & & & \\
\hline $\mathrm{HW}$ & 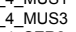 & Consuelo; Consuelo vein $H W$ atteration & $\begin{array}{l}n \\
\text { nalteracation } \\
\text { nation }\end{array}$ & iscovite after biotite & 46.53 & ${ }_{20.40}^{20.47}$ & 0.23 & 1.05 & 1.72 & & & 10.88 & & $\begin{array}{llll}89.82 & 6.58 & 4.90 & 0.02\end{array}$ & $\begin{array}{l}0.130 .39 \\
20.12\end{array}$ & & 1.9 & & 00 & 50 & 96 & 48 & & 69 & \\
\hline & 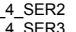 & onsuelo; Consuelo vein HW alteration & vein alteration & Idspar & $\begin{array}{l}48.80 \\
4790\end{array}$ & $\begin{array}{r}31.89 \\
22922\end{array}$ & & .08 & 1.37 & & & $\begin{array}{l}11.21 \\
11.13\end{array}$ & 031 & $\begin{array}{lll}94.46 & 6.54 & 5.04 \\
9920 & 662 & 4.76\end{array}$ & $\begin{array}{ll}0.12 & 0.27 \\
0.20 & 0.39\end{array}$ & & 1.92 & & & & & & & & \\
\hline & & $\begin{array}{l}\text { aleracuon } \\
\text { alteration }\end{array}$ & & ar & 47.21 & 3205 & & & $122-3$ & & & 11. & 0.31 & 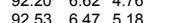 & 0. & & 1.940 .008 & & & & & & & & \\
\hline & SER2 & 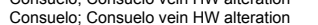 & & al & 48.20 & 30.40 & & 32 & 1.68 & & 0.26 & 10.26 & & 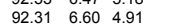 & $0.15 \mathrm{c}-10$ & 0.04 & 1.79 & & & & & & & & \\
\hline & SER5 & $\begin{array}{l}\text { Consuelo; Con } \\
\text { Cons }\end{array}$ & ation & dspar & 47.60 & 31.27 & & 1.23 & 1.29 & & & 10.82 & & $92.29 \quad 6.53 \quad 5.05$ & $0.14 \quad 0.26$ & & 1.89 & & & 1.58 & 89 & & & .59 & \\
\hline & & $\begin{array}{l}\text { Consuele; Cons } \\
\text { Consuelo: Cons }\end{array}$ & 'vein alteration & ricite after feldspar & ${ }_{46.52}^{46.52}-10$ & $\begin{array}{r}31.04 \\
33.83 \\
-10\end{array}$ & 0.22 & 1.32 & $\begin{array}{l}1.34 \\
1.81\end{array}$ & & & $\begin{array}{l}10 . \\
10 .\end{array}$ & 0.25 & $\begin{array}{r}91.08 \\
9526\end{array}$ & & & & & & & & & & & \\
\hline & $\begin{array}{l}\text { SER4 } \\
\text { SER }\end{array}$ & $\begin{array}{l}\text { Consuelor, ;o } \\
\text { Consul; Co }\end{array}$ & 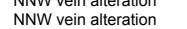 & 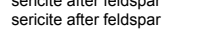 & $\begin{array}{l}49.14 \\
55.12\end{array}$ & $\begin{array}{l}37.96 \\
27.96\end{array}$ & & $\begin{array}{l}1.16 \\
.32\end{array}$ & 0.77 & & & 9.8 & 0.24 & $\begin{array}{l}4.98 \\
4.32\end{array}$ & 0.13 & & 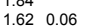 & & & & & & & & \\
\hline & SER5 & Consuelo; $\mathrm{CC}$ & tion & se & 55.73 & 25.26 & & 1. & 1.89 & & & 9.71 & & $93.89 \quad 7.39$ & 0. & & 1.64 & & .01 & 11.34 & 64 & 33 & & 75 & \\
\hline & SER2 & Consuelo; Consuelo vein wallrock clast & NWW ve & $\begin{array}{l}\text { sericte a } \\
\text { serite }\end{array}$ & 62.65 & $\begin{array}{l}19.31 \\
33032\end{array}$ & & 0. & 1.24 & & & $\begin{array}{l}7.45 \\
0.85\end{array}$ & & $\begin{array}{l}91.41 \\
0.18\end{array}$ & & & $\begin{array}{l}1.26 \\
171\end{array}$ & & & & 83 & & & & \\
\hline & & $\begin{array}{l}\text { ck clast } \\
\text { ck clast }\end{array}$ & & sericite & $\begin{array}{l}4.952 \\
554\end{array}$ & & & & 1.3 & & 0.84 & 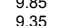 & & $\begin{array}{l}92.18 \\
93.49\end{array}-\mathrm{l} 2$ & & 0.12 & & & & & & & & & \\
\hline WR & SER5 & $\begin{array}{l}\text { wallirock clast } \\
\text { wats }\end{array}$ & Nn & $\begin{array}{l}\text { lericite } \\
\text { serite }\end{array}$ & 46.90 & ${ }_{34.33}^{20.95}$ & & 1.08 & 0.72 & & & 11.14 & 0.22 & 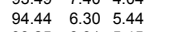 & 0.120 .14 & & 1.910 .06 & & 00 & 74 & 97 & 26 & 26 & 2.32 & \\
\hline & & wallrock clast & & & 46.41 & 10 & & 103 & 1 & & & 11.28 & 0.23 & 93.25 & 09 & & 1.960 .06 & & 99 & & & & & 32 & \\
\hline & $\begin{array}{l}\text { SER1 } \\
\text { SRR2 }\end{array}$ & $\begin{array}{l}\text { Papaga } \\
\text { Papaga }\end{array}$ & & & $\begin{array}{l}47.31 \\
4002\end{array}$ & 10 & $\begin{array}{l}0.26 \\
0.30\end{array}$ & 1.7. & $\begin{array}{l}1.41 \\
1.35\end{array}$ & & & $\begin{array}{l}10.61 \\
1101\end{array}$ & & $\begin{array}{l}91.41 \\
9550\end{array}$ & $\begin{array}{l}30 . \\
3\end{array}$ & & $\begin{array}{l}1.88 \\
1.87\end{array}$ & & & & & & & & \\
\hline & SER3 & $\begin{array}{l}\text { Papagayo; } \mathrm{G} \\
\text { Papage }\end{array}$ & & & $\begin{array}{l}48.62 \\
48.60\end{array}$ & 30.39 & 0.41 & 1.8 & 1.44 & & & 10.96 & & $\begin{array}{l}93.78 \\
93.78\end{array}$ & & & . & & & & & & & & \\
\hline & SER4 & Papagayo; Glo & 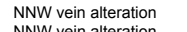 & par & 49.24 & 29.78 & 0.49 & 1.87 & 1.50 & & & 10.68 & & $\begin{array}{llll}93.45 & 6.69 & 4.77 & 0.05\end{array}$ & 50.210 .30 & & 1.85 & & & & & & & & \\
\hline & $\begin{array}{l}\text { SER1 } \\
\text { SER2 }\end{array}$ & & & & $\begin{array}{l}48.12 \\
483\end{array}$ & $\begin{array}{r}31.24 \\
33.36\end{array}-\mathrm{l}-\mathrm{l}$ & & & $\begin{array}{l}1.56 \\
1.35\end{array}$ & & & $\begin{array}{l}11.26 \\
1046\end{array}$ & 0.25 & $\begin{array}{l}93.74 \\
99.32\end{array}$ & & & $\begin{array}{l}1.95 \mathrm{C} \\
1.83\end{array}$ & & & & & & & & \\
\hline & & & & & & & & & $1.3 \times 340$ & & & & & & & & & & & & & & & & \\
\hline$F Y$ & SeR4 4 & 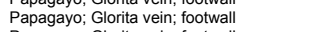 & Iteration & par & 50.15 & 31.63 & & 1.42 & 1.55 & & & 10.82 & & $95.75 \quad 6.63$ & 0.16 & & 1.83 & & & & 83 & & & 70 & \\
\hline & $\begin{array}{l}\text { SER1 } \\
\text { SER3 }\end{array}$ & & & & $\begin{array}{l}48.47 \\
48.43\end{array}$ & $\begin{array}{l}32.02 \\
29.65\end{array}$ & & 65 & $\begin{array}{l}1.35 \\
1.47\end{array}$ & & & $\begin{array}{l}11.24 \\
10.47\end{array}$ & 0.22 & $\begin{array}{l}94 \\
92\end{array}$ & & & & & & & & & & & \\
\hline & & & & & 48.59 & 30.46 & & 1.35 & 1.47 & & & 10 & & & & & & & & & & & & & \\
\hline & & & & & 45.90 & 29.32 & & 1.21 & 1.53 & & & & & 88 & & & & & & & & & & & \\
\hline & & & & & 45 & 20.40 & 0.21 & & 1.48 & & & 10. & 0.21 & 00 & & & & & & & & & & & \\
\hline & SER3 3 & & & & & 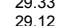 & 0.2 & 1. & 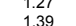 & & 0.15 & & & & & 0.02 & 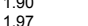 & & & & & & & & \\
\hline & 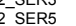 & & & & 45 & 2009.94 & 0.20 & & 1.34 & & & 10. & & & & & & & & & & & & & 8 \\
\hline & MUS2 & Glorita; GI & tion & sericite after biotite & 47.77 & 30.99 & 0.33 & 1.57 & 1.44 & & & & & & & & & & & & & & & & \\
\hline & & & & & & & & & & 025 & & & & & & & & & & & & & & & \\
\hline & 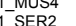 & $\begin{array}{l}\text { Glor } \\
\text { Glor }\end{array}$ & $\mathrm{NT}$ & sericite & $\begin{array}{l}46.90 \\
45.76\end{array}$ & 30.92 & $\begin{array}{l}0.18 \\
0.17\end{array}$ & & $\begin{array}{l}1.45 \\
1.54\end{array}$ & & & 10 & & $\begin{array}{l}3.02 \\
1002\end{array}$ & & & & & & & & & & & \\
\hline & 1_SER3 & Glorita; G & NNW v & $\begin{array}{l}\text { sernete } \\
\text { sericite }\end{array}$ & $\begin{array}{l}45.72 \\
45.10\end{array}$ & $\begin{array}{l}29.99 \\
29.99\end{array}$ & & 1.45 & $\begin{array}{l}1.44 \\
1.41\end{array}$ & & & 10.67 & 0.23 & $\begin{array}{lll}89.31 & 6.51 & 5.03\end{array}$ & 0.1 & & 1.940 .06 & & & & .00 & & & 2.59 & \\
\hline & 1. SER4 & Glori & & sericitit & 45.77 & $\begin{array}{l}29.21 \\
20.65\end{array}$ & & 1. & 1.45 & & & 10. & & 89.04 & & & 1.96 & & & & 96 & 19 & & 66 & 0 \\
\hline & SER1 & & & & & & & & 1.9 & & & & & & & & & & & & & & & & 42 \\
\hline & SER & & & & & 28.84 & 0.17 & & 1.75 & & & & & & & & & & & & & & & & \\
\hline & SER2 & Pap & vein ir & fg sericite & 48.03 & 31.03 & 0.19 & 2. & 1.55 & & & 11.62 & & 95.68 & 20. & & 2.0 & & U & & .08 & & & 然 & \\
\hline & SER & Pap & & $\begin{array}{l}\text { fg ser } \\
\text { fo ser }\end{array}$ & $\begin{array}{l}53.83 \\
4499\end{array}$ & $\begin{array}{l}26.25 \\
2921\end{array}$ & $\begin{array}{l}0.17 \\
0.15\end{array}$ & $\begin{array}{l}2.62 \\
2.61\end{array}$ & $\begin{array}{l}1.48 \\
1.55\end{array}$ & & & & & $\begin{array}{l}94.64 \\
8990\end{array}$ & & & & & & & & & & & \\
\hline & 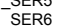 & $\begin{array}{l}\text { Pap } \\
\text { Pap }\end{array}$ & & fg sericite w & $\begin{array}{l}44.99 \\
45.70\end{array}$ & 29.21 & & $\begin{array}{l}2.61 \\
3.15\end{array}$ & 1.55 & & & 11. & & $\begin{array}{ll}89.90 & 6.73 \\
91.05 & 6.78 \\
\end{array}$ & $\begin{array}{rl}0 & 0.33 \\
0.39 & 0.35 \\
0.36\end{array}$ & & & & 23.00 & $\begin{array}{l}11.90 \\
11.80\end{array}$ & 19 & & & $\begin{array}{l}2.02 \\
2.71\end{array}$ & $\begin{array}{l}1 \\
3 \\
\end{array}$ \\
\hline S & 1.SER & Papa & veir & $\begin{array}{l}\text { fg sericite } \\
\text { f }\end{array}$ & 49.17 & 34 & & 0.89 & 0.58 & & $021+2-3$ & 10 & & & & 0 & & & & & 84 & & & 43 & 34 \\
\hline & SEF & & & g sericite & 47.46 & 32. & & & 1.2 & & 0 & 10 & 0. & & & & & & & & & & & & ? \\
\hline & SER1 & & & & & & & & & & & & & & & & & & & & & & & & \\
\hline & ${ }_{3}^{-}$SER 2 & & & tg seri & 47.94 & 33.06 & & 0.79 & 0.80 & & 0.24 & 10.63 & & 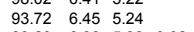 & 0.090 .16 & 0.03 & 1.83 & 13.8 & 22.01 & 11. & 86 & 25 & 0.25 & 47 & 35 \\
\hline & & & vein & fg serit & 49.57 & 33.22 & & 1.79 & 0.92 & & 0.18 & 10.27 & & $\begin{array}{lll}96.10 & 6.51 \quad 5.14\end{array}$ & $\begin{array}{ll}0.20 & 0.18 \\
\end{array}$ & 0.02 & & 13.77 & 22.0 & 11.6 & 1.74 & .38 & 0.38 & 2.54 & \\
\hline
\end{tabular}


Table 5 Average and range of selected elements from 8 whole rock geochemical samples of granodiorite from the Pataz district.

\begin{tabular}{lccc}
\hline Element & Average & Range & LLD $^{*}$ \\
\hline $\mathrm{K}_{2} \mathrm{O}(\%)$ & 2.55 & 2.25 to 3.19 & 0.01 \\
$\mathrm{Al}_{2} \mathrm{O}_{3}(\%)$ & 15.23 & 14.46 to 15.72 & 0.01 \\
$\mathrm{Au}(\mathrm{ppb})$ & 1 & $<1$ to 5 & 1 \\
$\mathrm{Cu}(\mathrm{ppm})$ & 4.9 & 2 to 10 & 1 \\
$\mathrm{~Pb}(\mathrm{ppm})$ & 12.4 & 8 to 16 & 5 \\
$\mathrm{Bi}(\mathrm{ppm})$ & $<2$ & $<2$ only & 2 \\
$\mathrm{Mn}(\%)$ & 0.08 & 0.06 to 1.00 & 0.001 \\
$\mathrm{Sb}(\mathrm{ppm})$ & 0.66 & 0.3 to 1.0 & 0.1 \\
$\mathrm{~W}(\mathrm{ppm})$ & 0.5 & $<1$ to 3 & 1 \\
$\mathrm{As}(\mathrm{ppm})$ & 2.4 & 1 to 4 & 1 \\
$\mathrm{Rb}(\mathrm{ppm})$ & 100 & 70 to 120 & 10 \\
$\mathrm{Te}(\mathrm{ppm})$ & n.d. & n.d. & \\
$\mathrm{Tl}(\mathrm{ppm})$ & n.d. & n.d. & \\
$3 \mathrm{~K} / \mathrm{Al}$ & 0.55 & 0.49 to 0.72 & \\
$(\mathrm{Rb}+\mathrm{Cs} / \mathrm{Th})_{\mathrm{N}}$ & 1.57 & 0.86 to 2.07 & \\
\hline * $\mathrm{LLD}=$ lower level of detection & n.d. no data
\end{tabular}

